\title{
ON SETS OF INTEGERS WHICH CONTAIN NO THREE TERMS IN GEOMETRIC PROGRESSION
}

\begin{abstract}
NATHAN MCNEW
Abstract. The problem of looking for subsets of the natural numbers which contain no three-term arithmetic progressions has a rich history. Roth's theorem famously shows that any such subset cannot have positive upper density. In contrast, Rankin in 1960 suggested looking at subsets without three-term geometric progressions, and constructed such a subset with density about 0.719. More recently, several authors have found upper bounds for the upper density of such sets. We significantly improve upon these bounds, and demonstrate a method of constructing sets with a greater upper density than Rankin's set. This construction is optimal in the sense that our method gives a way of effectively computing the greatest possible upper density of a geometricprogression-free set. We also show that geometric progressions in $\mathbb{Z} / n \mathbb{Z}$ behave more like Roth's theorem in that one cannot take any fixed positive proportion of the integers modulo a sufficiently large value of $n$ while avoiding geometric progressions.
\end{abstract}

\section{BACKGROUND}

Let $A$ be a subset of the positive integers. A three-term arithmetic progression in $A$ is a progression $(a, a+b, a+2 b) \in A^{3}$ with $b>0$, or equivalently, a solution to the equation $a+c=2 b$ where $a, b$ and $c$ are distinct elements of $A$. We say that $A$ is free of arithmetic progressions if it contains no such progressions. For any subset $A$ of the positive integers we denote by $d(A)$ its asymptotic density (if it exists) and its upper density by $\bar{d}(A)$.

In 1952 Roth proved the following famous theorem [16].

Theorem 1.1 (Roth). If $A$ is a subset of the positive integers with $\bar{d}(A)>0$, then A contains a three-term arithmetic progression.

In particular, Roth showed that for any fixed $\alpha>0$ and sufficiently large $N$, any subset of the integers $\{1, \ldots, N\}$ of size at least $\alpha N$ contains a three-term arithmetic progression. We can also view Roth's result as a statement about arithmetic progressions (with 3 distinct elements) in the group of integers modulo $N$. Namely, if we denote by $D(\mathbb{Z} / N \mathbb{Z})$ the size of the largest subset of $\mathbb{Z} / N \mathbb{Z}$ free of arithmetic progressions, Roth's argument can be used to show [17] $D(\mathbb{Z} / N \mathbb{Z})=O\left(\frac{N}{\log \log N}\right)$. This result has been improved several times, (see [9], 4], 19]) with the current best result, due to Sanders [18] (see also [3]),

$$
D(\mathbb{Z} / N \mathbb{Z})=O\left(\frac{N(\log \log N)^{5}}{\log N}\right) .
$$

Received by the editor October 8, 2013 and, in revised form, March 1, 2014.

2010 Mathematics Subject Classification. Primary 11B05, 11B75, 11Y60, 05D10. 
Roth's theorem has since been generalized by Szemerédi to progressions of arbitrary length.

Arithmetic-progression-free sets have also been studied in the context of arbitrary abelian groups. Meshulam [11] generalized Roth's theorem to finite abelian groups of odd order, and recently Lev [10] has extended Meshulam's ideas to arbitrary finite abelian groups, a result which will be needed later. Let $D(G)$ be the size of the largest subset of the finite group $G$ free of three-term arithmetic progressions. If 2 happens to divide $|G|$, it is possible to find examples of arithmetic progressions with repeated terms by looking at elements of order 2 , so we insist that our arithmetic progressions consist of 3 distinct elements. We also denote by $c(G)$ the number of components of the abelian group $G$ when written in the invariant factor decomposition $G \cong \mathbb{Z} / k_{1} \mathbb{Z} \times \mathbb{Z} / k_{2} \mathbb{Z} \times \cdots \times \mathbb{Z} / k_{t} \mathbb{Z}$ where $k_{1}\left|k_{2}\right| \cdots \mid k_{t}$, and write $2 G$ for the group of doubles $\{g+g: g \in G\}$.

Theorem 1.2 (Lev). For any abelian group $G, D(G)$ satisfies

$$
D(G) \leq \frac{2|G|}{c(2 G)}
$$

Combining Lev's theorem with Sanders' bound as in [11, Corollary 1.3] one obtains

Corollary 1.3. For any abelian group $G$ for which $c(G)=c(2 G)$ we have

$$
D(G) \ll \frac{|G|(\log \log |G|)^{5}}{(\log |G|)^{\frac{1}{2}}} .
$$

In a completely analogous manner, one can consider geometric progressions of integers of the form $\left(a, a k, a k^{2}\right)$ with $k \in \mathbb{Q}$ and $k>1$ (equivalently, a solution to the equation $a c=b^{2}$ with distinct integers $\left.a, b, c\right)$ and seek sets of integers which are free of such progressions. It is somewhat surprising just how different the results are in this case. We can immediately see, for example, that the squarefree integers, a set with asymptotic density $\frac{6}{\pi^{2}} \approx 0.61$, is free of geometric progressions.

Unlike the difference of two terms in an arithmetic progression, the ratio between successive terms of a geometric progression of integers need not be an integer. For example, the progression $(4,6,9)$ is a geometric progression with common ratio $\frac{3}{2}$. While most of the existing literature on this problem is concerned only with the rational-ratio case, we will also consider the problem restricted to integral ratios in the results that follow.

The problem of finding sets of integers free of geometric progressions was first considered by Rankin [14 who showed

Theorem 1.4. There exists a set, $G_{3}^{*}$, free of geometric progressions with asymptotic density $d\left(G_{3}^{*}\right)=\frac{1}{\zeta(2)} \prod_{i>0} \frac{\zeta\left(3^{i}\right)}{\zeta\left(2 \cdot 3^{i}\right)} \approx 0.719745$. (Here $\zeta$ is the Riemann zeta function.)

Since then, several papers have further investigated the problem of finding the largest possible sets which are geometric-progression-free. We will use the notation

$$
\begin{aligned}
& \bar{\alpha}=\sup \{\bar{d}(A): A \subset \mathbb{N} \text { is free of geometric progressions }\} \\
& \alpha=\sup \{d(A): A \subset \mathbb{N} \text { is free of geometric progressions and } d(A) \text { exists }\}
\end{aligned}
$$


for sets free of geometric progressions with rational ratio and

$\bar{\beta}=\sup \{\bar{d}(A): A \subset \mathbb{N}$ is free of integral ratio geometric progressions $\}$,

$\beta=\sup \{d(A): A \subset \mathbb{N}$ is free of integral ratio geometric progressions

$$
\text { and } d(A) \text { exists }\}
$$

for the corresponding values when we restrict the problem to integral ratios.

Clearly $\alpha \leq \bar{\alpha} \leq \bar{\beta}$ and $\alpha \leq \beta$. Rankin's construction remains the best lower bound for $\alpha$, and was, prior to this work, also the best lower bound for both $\bar{\alpha}$ and $\beta$, though Beiglböck, Bergelson, Hindman, and Strauss [1] had shown that $\bar{\beta} \geq 0.75$. More progress had been made on the upper bounds for $\bar{\alpha}$. Riddell [15] showed that $\bar{\alpha} \leq 6 / 7$. This bound was reproved by Beiglböck et al. [1], who were unaware of Riddell's result. Their result was an improvement of a bound obtained by Brown and Gordon [5] who, also unaware of Riddell's result, had shown that $\bar{\alpha}<0.86889$. Nathanson and O'Bryant 13 combined these methods to show that $\bar{\alpha}<0.84948$.

While the existing literature on geometric-progression-free sets has worked in greater generality, considering geometric progressions of length $k \geq 3$, we have chosen in this paper to focus on progressions of length 3 . While most of the methods and constructions in this paper generalize to arbitrary $k$, they don't, in general, appear to lead to a closed form expression in terms of $k$. We look first at the problem of finding geometric progressions of the residues modulo $n$ in Section 2 and find that for large enough $n$ any positive proportion of such residues will contain a geometric progression.

Section 3 considers sets of integers free of progressions with integral ratios and shows that one can construct such sets with substantially higher upper density than Rankin's set.

Our main results are in Section 4, where we demonstrate an algorithm for effectively computing the value of $\bar{\alpha}$. We use this method to significantly improve several of the best known bounds for the constants defined above. Namely we show that

$$
\begin{aligned}
& 0.730027<\bar{\alpha}<0.772059, \\
& 0.818410<\bar{\beta}<0.819222,
\end{aligned}
$$

and that

$$
0.722130<\beta .
$$

Finally, in Section 6 we investigate bounds for sets which have an asymptotic density.

\section{Geometric progressions in $\mathbb{Z} / n \mathbb{Z}$}

Rankin showed, in contrast to the arithmetic progression case, that one can take sets of integers with positive density (even the majority of integers) and still avoid geometric progressions. In light of Rankin's result, it is somewhat surprising that this does not remain true when we look at the integers modulo $n$. First, we need a proposition and a corollary of Lev's theorem for unit groups $(\mathbb{Z} / n \mathbb{Z})^{\times}$.

Proposition 2.1. Given any subgroup $H$ of an abelian group $G$,

$$
D(G) \leq \frac{|G| D(H)}{|H|} .
$$


Proof. If $A \subset G$ is free of arithmetic progressions, then for every coset $x H$ the set $A \cap x H$ is free of arithmetic progressions, and by the pigeonhole principle there exists at least one coset with

$$
|A \cap x H|=\left|x^{-1} A \cap H\right| \geq \frac{|A||H|}{|G|} .
$$

Thus, since $\left(x^{-1} A\right) \cap H$ is a subset of $H$ free of arithmetic progressions,

$$
|A| \leq \frac{|G|\left|\left(x^{-1} A\right) \cap H\right|}{|H|} \leq \frac{|G| D(H)}{|H|} .
$$

Corollary 2.2. For any integer $n$, the group $(\mathbb{Z} / n \mathbb{Z})^{\times}$of units modulo $n$ satisfies

$$
D\left((\mathbb{Z} / n \mathbb{Z})^{\times}\right) \ll \frac{\varphi(n)(\log \log n)^{5}}{(\log n)^{\frac{1}{2}}} .
$$

Proof. Let $G=(\mathbb{Z} / n \mathbb{Z})^{\times}$so that $|G|=\varphi(n)$. Write $G=\mathbb{Z} / 2 \mathbb{Z} \times \cdots \times \mathbb{Z} / 2 \mathbb{Z} \times H$ where $H$ is free of copies of $\mathbb{Z} / 2 \mathbb{Z}$ in its invariant factor decomposition, and hence $c(H)=c(2 H)$. Let $n=p_{1}^{k_{1}} p_{2}^{k_{2}} \cdots p_{\omega(n)}^{k_{\omega(n)}}$ be the prime factorization of $n$. Since

$$
(\mathbb{Z} / n \mathbb{Z})^{\times} \cong\left(\mathbb{Z} / p_{1}^{k_{1}} \mathbb{Z}\right)^{\times} \times\left(\mathbb{Z} / p_{2}^{k_{2}} \mathbb{Z}\right)^{\times} \times \cdots \times\left(\mathbb{Z} / p_{\omega(n)}^{k_{\omega}(n)} \mathbb{Z}\right)^{\times}
$$

and each group $\left(\mathbb{Z} / p_{j}^{k_{j}} \mathbb{Z}\right)^{\times}$contains at most one copy of $\mathbb{Z} / 2 \mathbb{Z}$ when $p_{j} \neq 2$ and at most two if $p_{j}=2$, we find that $G$ has at most $\omega(n)+1$ copies of $\mathbb{Z} / 2 \mathbb{Z}$. Thus the subgroup $H$ has size at least $\frac{\varphi(n)}{2^{\omega(n)+1}}$. Thus, using Corollary 1.3 and Proposition 2.1 ,

$$
\begin{aligned}
D(G) \leq \frac{|G| D(H)}{|H|} & \ll \frac{|G||H|(\log \log |H|)^{5}}{|H|(\log |H|)^{\frac{1}{2}}} \\
& \leq \frac{\varphi(n)(\log \log n)^{5}}{\left(\log \left(\frac{\varphi(n)}{2^{\omega(n)+1}}\right)\right)^{\frac{1}{2}}} .
\end{aligned}
$$

Using the facts [8, Theorem 323 and Section 22.10] that $\lim \inf \frac{\varphi(n) \log \log n}{n}=e^{-\gamma}$ and $\omega(n)=O\left(\frac{\log n}{\log \log n}\right)$, we have

$$
\begin{aligned}
\log \left(\varphi(n) / 2^{\omega(n)+1}\right) & \geq \log \left(\frac{n}{e^{\gamma} \log \log n}(1+o(1))\right)-(\omega(n)+1) \log (2) \\
& =\log n+O\left(\frac{\log n}{\log \log n}\right)
\end{aligned}
$$

So

$$
D(G) \ll \frac{\varphi(n)(\log \log n)^{5}}{(\log n)^{\frac{1}{2}}} .
$$

Theorem 2.3. Let $E(\mathbb{Z} / n \mathbb{Z})$ denote the size of the largest possible subset of the residues modulo $n$ which does not contain a three-term geometric progression. Then

$$
E(\mathbb{Z} / n \mathbb{Z}) \ll \frac{n(\log \log n)^{5}}{(\log n)^{1 / 2}} .
$$


Proof. For each $d \mid n$ let $R_{d}$ denote the set of $m \in \mathbb{Z} / n \mathbb{Z}$ such that $(m, n)=d$. So, for example, when $d=1, R_{1}=(\mathbb{Z} / n \mathbb{Z})^{\times}$. Note that $R_{d}$ can be viewed as $d\left(\mathbb{Z} / \frac{n}{d} \mathbb{Z}\right)^{\times}$ in the sense that each element of $R_{d}$ is uniquely representable as $d$ times a residue coprime to $\frac{n}{d}$. Thus $\left|R_{d}\right|=\varphi\left(\frac{n}{d}\right)$.

Furthermore, any "arithmetic" progression, $\left(a, a b, a b^{2}\right)$, (written multiplicatively) in the group $\left(\mathbb{Z} / \frac{n}{d} \mathbb{Z}\right)^{\times}$corresponds to the "geometric" progression $\left(d a, d a b, d a b^{2}\right)$ contained in the set of residues $R_{d}$. So any geometric-progression-free subset of $R_{d}$ cannot be larger than $D\left(\left(\mathbb{Z} / \frac{n}{d} \mathbb{Z}\right)^{\times}\right)$. Because the $R_{d}$ partition $\mathbb{Z} / n \mathbb{Z}$, we see that

$$
E(\mathbb{Z} / n \mathbb{Z}) \leq \sum_{d \mid n} D\left((\mathbb{Z} / d \mathbb{Z})^{\times}\right)=\sum_{\substack{d \mid n \\ d<\sqrt{n}}} D\left((\mathbb{Z} / d \mathbb{Z})^{\times}\right)+\sum_{\substack{d \mid n \\ d \geq \sqrt{n}}} D\left((\mathbb{Z} / d \mathbb{Z})^{\times}\right)
$$

$$
\begin{aligned}
& \ll \sum_{\substack{d \mid n \\
d<\sqrt{n}}} \sqrt{n}+\sum_{\substack{d \mid n \\
d \geq \sqrt{n}}} \frac{\varphi(d)(\log \log d)^{5}}{(\log d)^{\frac{1}{2}}} \\
& <\sum_{d \mid n} \sqrt{n}+\frac{(\log \log n)^{5}}{\left(\frac{1}{2} \log n\right)^{\frac{1}{2}}} \sum_{d \mid n} \varphi(d) \\
& \ll n^{1 / 2+\epsilon}+\frac{n(\log \log n)^{5}}{(\log n)^{\frac{1}{2}}} \ll \frac{n(\log \log n)^{5}}{(\log n)^{\frac{1}{2}}} .
\end{aligned}
$$

Where we used the fact that the number of divisors of $n$ is $O\left(n^{\epsilon}\right)$ for every $\epsilon>0$.

Bounding the exponent on $\log n$ in this theorem is complicated by the fact that the size, $\lambda(n)$, of the largest cyclic subgroup of the unit group $(\mathbb{Z} / n \mathbb{Z})^{\times}$can occasionally be very small. In general, however, this is not the case. In fact, we know [6. Lemma 2] that there exists a set $S$ of integers with asymptotic density 1 such that for all $n$ in $S, \lambda(n)=n /(\log n)^{\log \log \log n+O(1)}$.

Let $n \in S$ and let $H$ be a cyclic subgroup of $(\mathbb{Z} / n \mathbb{Z})^{\times}$of size $\lambda(n)$. Then, using Sanders bound (11) and Proposition 2.1 we have

$$
\begin{aligned}
D\left((\mathbb{Z} / n \mathbb{Z})^{\times}\right) & \leq \frac{\left|(\mathbb{Z} / n \mathbb{Z})^{\times}\right| D(H)}{|H|} \\
& \ll \frac{\varphi(N)|H|(\log \log |H|)^{5}}{|H| \log |H|} \\
& =\frac{\varphi(n)\left(\log \log \left(n /(\log n)^{\log \log \log n+O(1)}\right)\right)^{5}}{\log \left(n /(\log n)^{\log \log \log n+O(1)}\right)} \\
& \ll \frac{\varphi(n)(\log \log n)^{5}}{\log n} .
\end{aligned}
$$

We know [7, Lemma 2] that for each $d \mid n$,

$$
\frac{\lambda(d)}{d} \geq \frac{\lambda(n)}{n}
$$

so for $n \in S$, and $d \mid n, \lambda(d) \geq \frac{d}{(\log n)^{\log \log \log n+O(1)}}$. If we assume $d>\sqrt{n}$, then the above argument shows that $D\left((\mathbb{Z} / d \mathbb{Z})^{\times}\right) \ll \frac{\varphi(d)(\log \log d)^{5}}{\log d}$. Using this inequality in place of Corollary 2.2 in (2) of the proof above, we have

Theorem 2.4. For $n \in S, E(\mathbb{Z} / n \mathbb{Z}) \ll \frac{n(\log \log n)^{5}}{\log n}$. 


\section{Geometric progressions With integral Ratio}

As mentioned before, it is possible to consider geometric progressions of two different types, depending on whether or not the ratio common to the progression is an integer. One would expect that restricting to the integral case should allow us to construct sets with larger asymptotic density, since there are fewer restrictions on our set. While Rankin's set $G_{3}^{*}$ is constructed to avoid rational geometric progressions, no integers can be added to it without introducing an integer geometric progression. Nevertheless, we can construct sets with substantially higher upper density. The following construction extends the method described in [1, Theorem $3.2]$.

Theorem 3.1. We have the lower bound $\bar{\beta}>0.815509$.

Proof. Note that for any $N$, the set $\left(\frac{N}{4}, N\right]$ is free of geometric progressions (with integral ratio) since for any $n \in\left(\frac{N}{4}, N\right]$ and $r \geq 2$, we have $n r^{2}>N$. This observation, combined with the argument described below, could be used to construct a set with upper density $\frac{3}{4}$. We can, however, do better.

Rather than just using the range $\left(\frac{N}{4}, N\right]$, we note that the set $\left(\frac{N}{9}, \frac{N}{8}\right] \cup\left(\frac{N}{4}, N\right]$ also has the property of being free of geometric progressions since for any $n \in$ $\left(\frac{N}{9}, \frac{N}{8}\right]$, the integer $2 n$ lies in the omitted interval $\left(\frac{N}{8}, \frac{N}{4}\right]$, hence $n$ is not part of a geometric progression with common ratio 2 , and $9 n>N$, meaning $n$ cannot be part of a progression of common ratio greater than or equal to 3 . One can further check that the set

$$
\mathbb{S}_{N}=\left(\frac{N}{48}, \frac{N}{45}\right] \cup\left(\frac{N}{40}, \frac{N}{36}\right] \cup\left(\frac{N}{32}, \frac{N}{27}\right] \cup\left(\frac{N}{24}, \frac{N}{12}\right] \cup\left(\frac{N}{9}, \frac{N}{8}\right] \cup\left(\frac{N}{4}, N\right]
$$

has this property, and for sufficiently large $N$ will contain at least

$$
\frac{3523 N}{4320}-6>0.815509 N
$$

integers less than $N$. (We can continue this process, adding smaller and smaller intervals to $\mathbb{S}_{N}$ indefinitely, and create sets with marginally greater density. However, we cannot take another such interval until $N / 2208$.)

Now, fix $N=N_{1}$, and let $N_{2}=48^{2} N_{1}$. The set $\mathbb{S}_{N_{1}} \cup \mathbb{S}_{N_{2}}$ will also be free of geometric progressions with integral ratio, since if $n, n r \in \mathbb{S}_{N_{1}}$ then $r<48$ so $n r^{2}<48 N_{1}=N_{2} / 48$, and so $n r^{2} \notin \mathbb{S}_{N_{2}}$. Similarly, if $n r, n r^{2} \in \mathbb{S}_{N_{2}}$, we again have $r<48$ and so

$$
n>\frac{n r}{48}>\frac{N_{2}}{48^{2}}=N_{1},
$$

thus $n \notin \mathbb{S}_{N_{1}}$. In general, set

$$
N_{i}=\frac{48^{2} N_{i-1}^{2}}{N_{1}}
$$

Then any geometric progression with two elements contained in $\mathbb{S}_{N_{i}}$ will not have a third element in the union of the $\mathbb{S}_{N_{j}}$. Furthermore, if $n \in \mathbb{S}_{N_{i}}$ and $n r \in \mathbb{S}_{N_{j}}$ for some $j>i$, then $r<\frac{48 N_{j}}{N_{i}}<\frac{48 N_{j}}{N_{1}}$, so $n r^{2}<\frac{48 N_{j}^{2}}{N_{1}}=\frac{N_{j+1}}{48}$ and so $n r^{2} \notin \mathbb{S}_{N_{k}}$ for any $k \geq j$. As a result, no geometric progression can have terms in 3 distinct $\mathbb{S}_{N_{i}}$.

Thus, letting $\mathcal{S}$ be the union of all such $\mathbb{S}_{N_{i}}$, we find that $\bar{d}(\mathcal{S})>0.815509$, and the entire set $\mathcal{S}$ is free of geometric progressions with integral ratio. 
Kevin Ford points out that a slightly higher upper density can be achieved by letting

$$
\mathbb{S}_{N}^{\prime}=\left(\frac{N}{48}, \frac{N}{12}\right] \cup\left(\frac{N}{4}, N\right] \cup\left\{\frac{N}{9}<n \leq \frac{N}{8}: 3 \nmid n, 4 \nmid n, 5 \nmid n\right\} .
$$

By a similar argument this set is also free of geometric progressions with integral ratio. In this case any geometric progression which tries to "bridge the gap" between the intervals will have a term divisible by 3,4 or 5 in the middle interval. Setting $\mathbb{S}_{N}=\mathbb{S}_{N}^{\prime} \cup \mathbb{S}_{\frac{N}{48}}^{\prime}$ we see that $\mathbb{S}_{N}$ is free of geometric progressions since each of its components is, and any geometric progression with terms in both components would need to have ratio greater than 48 to jump the gap. Constructing $\mathcal{S}$ using widely separated copies of $\mathbb{S}_{N}$ as above we find that $\bar{d}(\mathcal{S})=\left(1+\frac{1}{48^{2}}\right) \frac{589}{720}>0.81841$, giving us the following stronger bound.

Theorem 3.2 (Ford). We have the better lower bound $\bar{\beta}>0.818410$.

We are also able to construct sets free of geometric progressions with integral ratio with a (slightly) higher asymptotic density than the set generated by the greedy algorithm, described by Rankin. For convenience we recall the proof of Theorem 1.4 which constructs Rankin's set, $G_{3}^{*}$.

Proof. Note that if $\left(a, a k, a k^{2}\right)$ is a geometric progression and we denote by $v_{p}(a)$ the $p$-adic valuation of $a$, then $\left(v_{p}(a), v_{p}(a k), v_{p}\left(a k^{2}\right)\right)$ forms an arithmetic progression (which is nontrivial if $v_{p}(k) \neq 0$ ). Thus, any set of integers, all of whose prime factors occur with exponent contained in a set $A$ free of arithmetic progressions, will be free of geometric progressions [5. Theorem 1]. Take $A=A_{3}^{*}=\{0,1,3,4,9, \ldots\}$ to be the set of nonnegative integers which do not have a digit two in their ternary expansions. This is the set obtained by choosing integers free of arithmetic progressions using a greedy algorithm. Now, letting $G_{3}^{*}=\left\{n \in \mathbb{N}\right.$ : for all primes $\left.p, v_{p}(n) \in A_{3}^{*}\right\}$, we obtain a set free of geometric progressions. Note that this set is also the set obtained using a greedy algorithm to choose positive integers free of geometric progressions, either of integer or rational ratio. [5]

The density of this set, $G_{3}^{*}$, can be found using an Euler product reminiscent of the Euler product for the density of squarefree numbers. For a fixed prime $p$ the asymptotic density of those integers divisible by an acceptable power of the prime $p$ is given by

$$
\begin{aligned}
\left(\frac{p-1}{p}\right)\left(\sum_{i \in A_{3}^{*}} \frac{1}{p^{i}}\right) & =\left(\frac{p-1}{p}\right)\left(1+\frac{1}{p}\right) \prod_{i>0}\left(1+\frac{1}{p^{3^{i}}}\right) \\
& =\left(1-\frac{1}{p^{2}}\right) \prod_{i>0}\left(1+\frac{1}{p^{3^{i}}}\right) .
\end{aligned}
$$

Then, by the Chinese remainder theorem, we can compute the density of $G_{3}^{*}$ as a product over all primes,

$$
d\left(G_{3}^{*}\right)=\prod_{p}\left(\left(1-\frac{1}{p^{2}}\right) \prod_{i>1}\left(1+\frac{1}{p^{3^{i}}}\right)\right)=\frac{1}{\zeta(2)} \prod_{i>0} \frac{\zeta\left(3^{i}\right)}{\zeta\left(2 \cdot 3^{i}\right)} \approx 0.719745 .
$$

So, for example, considering just the primes 2, 3 and 5, Rankin's construction would include numbers of the form $2 \cdot 3 \cdot 5 k$, where $k$ is an integer divisible only by primes larger than 5 , for each prime $p>5, v_{p}(k) \in A_{3}^{*}$. If, instead, we were to 
exclude integers of this form, we would be able to include numbers of the following forms:

$$
2^{2} \cdot 3 \cdot 5 k, 2 \cdot 3^{2} \cdot 5 k, 2 \cdot 3 \cdot 5^{2} k, 2^{2} \cdot 3^{2} \cdot 5 k, 2 \cdot 3^{2} \cdot 5^{2} k, 2^{2} \cdot 3 \cdot 5^{2} k \text { and } 2^{2} \cdot 3^{2} \cdot 5^{2} k \text {. }
$$

Each of these new numbers we have included will force us to exclude numbers with these primes to higher powers, but in the end (calculating these inclusion/exclusions with a computer) we find that we gain about 0.002385 asymptotically in the trade, producing a new set with asymptotic density about 0.722130 . This proves a new lower bound for the constant $\beta$, defined in Section 1

Theorem 3.3. We have the lower bound $\beta>0.722130$.

This can be further improved by incorporating more primes and exclusions. Note, however, that this larger set does include progressions with rational ratios. For example, progressions of the form $\left(2^{3} \cdot 5 k, 2^{2} \cdot 3 \cdot 5 k, 2 \cdot 3^{2} \cdot 5 k\right)$ are included even though they form a progression with common ratio $\frac{3}{2}$.

\section{UPPER BOUNDS}

Upper bounds for the densities of sets of integers free of geometric progressions have been studied in several papers, with the current best bound being that of Nathanson and O'Bryant, that the upper density of any geometric-progression-free set is at most 0.84948. (Riddell [15] gives the upper bound 0.8339, but states that: "The details are too lengthy to be included here.") In this section we improve this bound to 0.772059 .

We consider first the problem of avoiding geometric progressions with ratios involving only a finite set of primes, in particular, the primes smaller than some bound $s$. Denote by $g_{s}(N)$ the cardinality of the largest subset of the integers $\{1, \ldots, N\}$ which is free of three-term rational-ratio geometric progressions which have common ratio involving only the primes less than or equal to $s$. We will see in Theorem 4.2 that for any $s$ the $\operatorname{limit}_{\lim _{N \rightarrow \infty}} \frac{g_{s}(N)}{N}$ exists and furthermore is equal to the supremum of the upper densities of sets of positive integers which avoid such progressions. We will denote this value by

$\overline{\alpha_{s}}=\sup \{\bar{d}(A): A \subset \mathbb{N}$ is free of $s$-smooth rational geometric progressions $\}$.

The simplest case, $s=2$, in which all of the ratios involved are integral, was recently studied (along with sets avoiding geometric progressions whose ratios involve any one, single, integer) by Nathanson and O'Bryant 12. They show that $\overline{\alpha_{2}}$ is an irrational number approximately 0.846378 with error less than 0.000001 .

Before proving the result for general $s$, we consider the specific case of just the primes 2 and 3, which is illustrative of the general behavior when multiple primes are involved.

Claim 4.1. The $\operatorname{limit} \lim _{N \rightarrow \infty} \frac{g_{3}(N)}{N}$ exists and is equal to $\overline{\alpha_{3}}$. Furthermore, this quantity is bounded by

$$
0.790470<\overline{\alpha_{3}}<0.791266 .
$$

Proof. Fix $N>0$ and consider the largest subset of the integers $\{1, \ldots, N\}$ free of geometric progressions which have a common ratio involving only the primes 2 and 3 . Denote by $S_{k}^{(3)}$ the set of 3 -smooth numbers (numbers whose only prime divisors are 2 and 3 ) at most $k$. Note first that any geometric-progression-free subset of $S_{4}^{(3)}=\{1,2,3,4\}$ must exclude at least one integer from this set, and hence for 
any integer $b \leq \frac{N}{4}$ such that $(b, 6)=1$ our set must exclude at least one of the integers $b, 2 b, 3 b, 4 b$. (The single one excluded cannot be $3 b$.) Since these numbers are distinct for different values of $b$, and $\frac{1}{3}=\frac{\phi(6)}{6}$ of the integers less than $\frac{N}{4}$ are coprime to 6 , we must exclude a total of at least $\frac{1}{3}\left(\frac{N}{4}\right)+O(1)$ integers.

If we now consider $S_{9}^{(3)}=\{1,2,3,4,6,8,9\}$, we find that this set contains the 4 progressions $(1,2,4),(2,4,8),(1,3,9)$ and $(4,6,9)$ which cannot all be precluded by removing any single number. However, removing the two integers 2 and 9 suffices. This means that for each $b \leq \frac{N}{9},(b, 6)=1$, we must exclude at least two of the integers from the set $\{b, 2 b, 3 b, 4 b, 6 b, 8 b, 9 b\}$, and moreover these sets are disjoint, not only from each other, but also simply extend the sets constructed from $S_{4}^{(3)}$ above. Thus each $b \leq \frac{N}{9}$ with $(b, 6)=1$ corresponds to an additional excluded integer, meaning we must now exclude at least $\frac{1}{3}\left(\frac{N}{4}+\frac{N}{9}\right)+O(1)$ integers.

In general, for each $k$ we can find a (not necessarily unique) subset $T_{k}^{(3)} \subset S_{k}^{(3)}$ which is as large as possible while avoiding geometric progressions. For each value of $k$ at which the number of necessary exclusions, $m_{k}=\left|S_{k}^{(3)}\right|-\left|T_{k}^{(3)}\right|$ increases (a new exclusion is required) there are an additional $\frac{N}{3 k}$ integers which must be excluded from our set. One can check computationally that the first few values of $k$ which require an additional exclusion are given in the following table.

TABLE 1

\begin{tabular}{|c|c|c|c|c|c|}
\hline$k$ & $\begin{array}{c}\text { \# of integers } \\
\text { excluded from } S_{k}^{(3)}\end{array}$ & $k$ & $\begin{array}{c}\text { \# of integers } \\
\text { excluded from } S_{k}^{(3)}\end{array}$ & $k$ & $\begin{array}{c}\text { \# of integers } \\
\text { excluded from } S_{k}^{(3)}\end{array}$ \\
\hline 4 & 1 & 243 & 13 & 1458 & 25 \\
9 & 2 & 256 & 14 & 1728 & 26 \\
16 & 3 & 15 & 1944 & 27 \\
18 & 4 & 16 & 2048 & 28 \\
32 & 5 & 17 & 2304 & 29 \\
36 & 6 & 18 & 2592 & 30 \\
64 & 7 & 19 & 3072 & 31 \\
81 & 8 & 20 & 3888 & 32 \\
96 & 9 & 512 & 21 & 4096 & 33 \\
128 & 10 & 576 & 22 & 4374 & 34 \\
144 & 11 & & 19 & 5184 & 35 \\
192 & 12 & 1024 & 23 & 5832 & 36 \\
\hline
\end{tabular}

Taking all these exclusions into account, we find that we have excluded

$$
\frac{N}{3}\left(\frac{1}{4}+\frac{1}{9}+\frac{1}{16}+\frac{1}{18}+\frac{1}{32}+\cdots+\frac{1}{5832}\right)+O(1)
$$

numbers. Since $\frac{1}{3}\left(\frac{1}{4}+\frac{1}{9}+\cdots+\frac{1}{5832}\right)>0.208734$, we have that $g_{3}(N)<0.791266 N$ for $N$ sufficiently large. 
Note that the process described above is also constructive. For a fixed integer $N$ we can take, for each $b \leq N,(b, 6)=1$, the set of integers $b \cdot T_{f(b)}^{(3)}$ where

$$
f(b)= \begin{cases}\left\lfloor\frac{N}{b}\right\rfloor & b>\frac{N}{5832} \\ 5832 & \text { otherwise }\end{cases}
$$

and not include any progressions involving the primes 2 and 3. Taking the union over all such $b$, the set we construct will differ in size from our upper bound only by the trailing terms in the series of 3 -smooth numbers greater than 5832 which we have not yet taken into account. Since

$$
\sum_{\substack{n>5832 \\ n \text { is } 3 \text {-smooth }}} \frac{1}{3 n}<0.000795,
$$

we have that $g_{3}(N)>0.790470 N$. As we take into account more 3 -smooth numbers the trailing reciprocal sum of these numbers above will become arbitrarily small, so we see that the $\operatorname{limit}_{N \rightarrow \infty} \frac{g_{3}(N)}{N}$ exists.

We can now go one step further and use this idea to construct a set of positive integers which has this upper density. As in Theorem 3.1, we can piece together widely separated sets of integers each constructed by the method just described. The resulting set can have upper density arbitrarily close to the value above, while avoiding geometric progressions involving the primes 2 and 3 .

This construction therefore gives us a lower bound for $\overline{\alpha_{3}}$, the supremum of the upper densities of all such sets. Since $\overline{\alpha_{3}}$ must also satisfy the same upper bound as $\frac{g_{3}(N)}{N}$, we can conclude that in fact $\overline{\alpha_{3}}=\lim _{N \rightarrow \infty} \frac{g_{3}(N)}{N}$.

There is nothing particular about the primes 2 and 3 in this argument. In general, write the sequence of $s$-smooth numbers $1=n_{1}<n_{2}<\ldots$ in increasing order. For each $j$ let $T_{n_{j}}^{(s)}$ denote a subset of $S_{n_{j}}^{(s)}=\left\{n_{1}, n_{2}, \ldots, n_{j}\right\}$ which is as large as possible (though not necessarily unique) while avoiding triples in geometric progression, and let $m_{n_{j}}=\left|S_{n_{j}}^{(s)}\right|-\left|T_{n_{j}}^{(s)}\right|$. Note that the $m_{n_{j}}$ are nondecreasing. Let $I_{s}$ be the set of numbers $j$ with $m_{n_{j}}>m_{n_{j-1}}$ (those $j$ for which $S_{n_{j}}^{(s)}$ requires an additional exclusion). For $s=3$ these are the numbers appearing in Table 1. We have the following result.

Theorem 4.2. For each integer $s \geq 2$,

$$
\overline{\alpha_{s}}=1-\left(\prod_{p \leq s} \frac{p-1}{p}\right) \sum_{j \in I_{s}} \frac{1}{n_{j}} .
$$

Since any geometric-progression-free subset of the integers must be free of ratios that involve only the primes 2 and 3, we see that the upper bound above, 0.791266, is also an upper bound for $\bar{\alpha}$. Already this value is better than the bounds given before in the literature, but we can improve this result further.

If we consider now the primes 2,3 and 5 , we see that the proof above goes through in exactly the same way, requiring additional exclusions at each of the integers

$\begin{array}{rrrrrrrrrrrrr}4 & 9 & 16 & 18 & 20 & 25 & 32 & 36 & 50 & 60 & 64 & 75 & 80 \\ 96 & 100 & 108 & 128 & 144 & 150 & 160 & 192 & 200 & 225 & 240 & 243 & 256 \\ 300 & 320 & 324 & 384 & 400 & 432 & 480 & 500 & 512 & 540 & & & \end{array}$


which are the first 36 exclusions required. This list gives us the bounds $0.766512<$ $\overline{\alpha_{5}}<0.782571$. The difficulty in pushing this method further is the amount of computation required to find the largest geometric-progression-free subset of $S_{k}^{(5)}$ (the 5-smooth numbers up to $k$ ) for increasingly larger $k$. For example, showing that an additional exclusion is required at 576 (the next 5-smooth number after 540) would require showing that there are no geometric-progression-free subsets of size 36 among the 705 -smooth numbers up to 576 .

Even though computational limitations prevent us from finding the exact values where additional exclusions are necessary past 540 in $S_{k}^{(5)}$ we can still use some of the computational work we did to estimate $\overline{\alpha_{3}}$ to further improve the upper bound on $\overline{\alpha_{5}}$. Taking $I_{3}$ (the set of values where an additional exclusion was required to avoid 3-smooth rational-ratio geometric progressions among the 3 -smooth integers) and multiplying its elements by each power of 5 , we obtain a subset of the 5 -smooth numbers,

$$
J_{5,3}=\bigcup_{i \geq 0} 5^{i} \cdot I_{3}
$$

$J_{5,3}$ is the set of values at which an additional exclusion is required to avoid 3 -smooth rational-ratio progressions among the 5 -smooth integers. Since avoiding 5 -smooth progressions will only require more exclusions, we know that the $i$ th smallest entry of $J_{5,3}$ is greater than or equal to the $i$ th entry of $I_{5}$, and thus can be used as an upper bound for this value.

So, multiplying 4,9,16,18,32 .5832, the numbers from Table 1 (the first 36 elements of $I_{3}$ ) by $1,5,25,125 \ldots$ and reordering we obtain the first few entries of $J_{5,3}$ : (4)

$\begin{array}{rrrrrrrrrrrrrr}4 & 9 & 16 & 18 & 20 & 32 & 36 & 45 & 64 & 80 & 81 & 90 & 96 & \\ 100 & 128 & 144 & 160 & 180 & 192 & 225 & 243 & 256 & 288 & 320 & 384 & 400 & \\ 405 & 450 & 480 & 486 & 500 & 512 & 576 & 640 & 720 & 729 & 800 & 864 & 900 & \ldots\end{array}$

Note that each term in list (41) is greater than or equal to the corresponding term in (3). Looking at the 37th entry of this table we see that we will require an additional exclusion by the time we reach 800 . So, taking all of these exclusions into account (first the 36 exclusions from (3), and then those starting at 800 from (4)) we can decrease our bound by an additional 0.006815, so $\overline{\alpha_{5}}<0.775755$. Applying this process again for the primes $2,3,5$ and 7 , where we compute that exclusions must be made at

$$
\begin{array}{rrrrrrrrrrrrr}
4 & 9 & 16 & 18 & 20 & 25 & 28 & 32 & 36 & 49 & 50 & 60 & 64 \\
72 & 75 & 81 & 96 & 98 & 100 & 108 & 112 & 126 & 128 & 144 & 147 & 150
\end{array}
$$

and incorporating the exclusions calculated for both the 2, 3 and 2, 3, 5 cases, as described above, we obtain the bound $\overline{\alpha_{7}}<0.772059$. Again, this is an upper bound for the upper density of a set of integers avoiding all geometric progressions which proves the following.

Theorem 4.3. We have $\bar{\alpha}<0.772059$.

Since this upper bound is lower than the upper density of the set we constructed for the integer-ratio problem in Theorem 3.1, we see (as one might have expected) that these two problems, considering integer and rational ratios, are in fact different. One can carry through an analogous argument considering only progressions with 
integral ratios, in which case we find (looking at 3 -smooth integer progressions) that we must make exclusions at

$\begin{array}{rrrrrrrrrrrr}4 & 9 & 18 & 32 & 48 & 64 & 96 & 128 & 144 & 192 & 256 & 288 \\ 384 & 432 & 512 & 648 & 864 & 972 & 1024 & 1296 & 1536 & 1944 & 2187 & 2304 \\ 2916 & 3456 & 4096 & 4608 & 5832 & 6144 & 6912 & 8748 & 9216 & & & \end{array}$

yielding an upper bound of 0.820555 . If we combine this argument, as in the rational-ratio case above, with the necessary exclusions for 5 -smooth progressions,

$\begin{array}{rrrrrrrrrrrrr}4 & 9 & 18 & 20 & 32 & 40 & 48 & 64 & 80 & 96 & 100 & 128 & 144 \\ 160 & 192 & 200 & 240 & 256 & 288 & 320 & 384 & 400 & 432 & 400 & 432 & 480 \\ 500 & 512 & & & & & & & & & & & \end{array}$

we can compute a new upper bound for $\bar{\beta}$ which is less than 0.001 above the lower bound of the set constructed in Section 3 .

Theorem 4.4. We have $0.818410<\bar{\beta}<0.819222$.

We can also use the set we constructed in the proof of Claim 4.1 which had high upper density while avoiding geometric progressions involving the primes 2 and 3 , to construct sets free of any rational-ratio progression which have a higher upper density than Rankin's set.

Theorem 4.5. There exist geometric-progression-free sets with upper density greater than 0.730027 , so $0.730027<\bar{\alpha}<0.772059$.

Proof. Recall that Rankin's construction consisted of integers with exponents on primes contained in the set $A_{3}^{*}$, the greedily chosen set free of arithmetic progressions. To construct a set with greater upper density, we start with the set described in Claim 4.1, denoted here by $H_{3}$, which is free of geometric progressions involving the primes 2 and 3 . Recall that $\bar{d}\left(H_{3}\right)>0.790470$. We now remove from $H_{3}$ those integers which have a prime greater than 3 with exponent not contained in $A_{3}^{*}$. Symbolically we construct

$$
H_{3}^{\prime}=\left\{n \in H_{3}: \forall p \geq 5, v_{p}(n) \in A_{3}^{*}\right\} .
$$

Essentially, rather than taking all integers, $b$, coprime to 6 in the argument above, we use Rankin's construction to choose integers coprime to 6 which do not themselves contain any geometric progressions. We then have found a more efficient way (in regard to upper density) of choosing exponents for the primes 2 and 3 than Rankin's method.

To find the upper density of this set, we recall the Euler product of the density of Rankin's set,

$$
\frac{1}{\zeta(2)} \prod_{i>0} \frac{\zeta\left(3^{i}\right)}{\zeta\left(2 \cdot 3^{i}\right)}=\prod_{p}\left(\frac{p-1}{p} \sum_{i \in A_{3}^{*}} p^{-i}\right) .
$$

Now, fix an integer $N$ and consider, for example, the interval $[N / 6, N / 8)$. Since 4 required one exclusion and 6 did not yet require an additional exclusion, we could take for each $b \in[N / 6, N / 8)$ with $(b, 6)=1$ four of the five integers $b, 2 b, 3 b, 4 b, 6 b$ without introducing a 3 -smooth geometric progression, a total contribution of $\frac{4}{3}\left(\frac{N}{4}-\frac{N}{6}\right)+O(1)$ integers less than $N$. We now add the further restriction that our integers $b$ must be in the set $G_{3}^{*}$ (In order to avoid progressions involving other 
primes) as well as being coprime to 6 . Such candidates for $b$ have asymptotic density

$$
\frac{1}{3} \prod_{p \geq 5}\left(\frac{p-1}{p} \sum_{i \in A_{3}^{*}} p^{-i}\right)
$$

and so the contribution from the range $[N / 6, N / 8)$ is now

$$
\frac{4}{3}\left(\frac{N}{4}-\frac{N}{6}\right) \prod_{p \geq 5}\left(\frac{p-1}{p} \sum_{i \in A_{3}^{*}} p^{-i}\right)+O(1) .
$$

Doing this for every interval gives us a contribution of

$$
\left(1-\frac{N}{3}\left(\frac{1}{4}+\frac{1}{9}+\cdots+\frac{1}{5832}+\sum_{\substack{n>5832 \\ n \text { is } 3 \text {-smooth }}} \frac{1}{3 n}\right)\right) \prod_{p \geq 5}\left(\frac{p-1}{p} \sum_{i \in A_{3}^{*}} p^{-i}\right)+O(1),
$$

and so

$$
\bar{d}\left(H_{3}^{\prime}\right)>0.790470 \prod_{p \geq 5}\left(\frac{p-1}{p} \sum_{i \in A_{3}^{*}} p^{-i}\right)>0.730027
$$

\section{Computing $\bar{\alpha}$}

The arguments given above show, not only how to compute good approximations for each $\overline{\alpha_{s}}$ which we can use to bound $\bar{\alpha}$, but also that these values converge to $\bar{\alpha}$.

Theorem 5.1. In the limit, as a larger set of initial primes is taken into account, $\lim _{s \rightarrow \infty} \overline{\alpha_{s}}=\bar{\alpha}$.

Proof. The arguments of Theorems 4.5 and 4.2 show that

$$
\overline{\alpha_{s}} \prod_{p>s}\left(\frac{p-1}{p} \sum_{i \in A_{3}^{*}} p^{-i}\right) \leq \bar{\alpha} \leq \overline{\alpha_{s}}
$$

and since this Euler product converges, we know that

$$
\lim _{s \rightarrow \infty} \prod_{p>s}\left(\frac{p-1}{p} \sum_{i \in A_{3}^{*}} p^{-i}\right)=1 .
$$

The conclusion follows.

Thus, since we have a method to compute each $\overline{\alpha_{s}}$ to any desired precision, we can also do so for the constant $\bar{\alpha}$ by extending the methods described above. Here, we look at the complexity of computing $\bar{\alpha}$.

Theorem 5.2. For each number $\epsilon$ with $0<\epsilon<1$, the constant $\bar{\alpha}$ can be computed to within $\epsilon$ in time $O\left(1.6538^{\left(-2 \log _{2} \epsilon\right)^{\frac{1}{\epsilon}}}\right)$.

Proof. We need, first, to consider a sufficient number of primes so that

$$
\prod_{p>s}\left(\frac{p-1}{p} \sum_{i \in A_{3}^{*}} p^{-i}\right)>1-\epsilon / 2
$$


where $A_{3}^{*}$ is the greedily chosen set of integers free of arithmetic progressions used in Rankin's construction. Using the inequality

$$
\begin{aligned}
\prod_{p>s}\left(\frac{p-1}{p} \sum_{i \in A_{3}^{*}} p^{-i}\right) & >\prod_{p>s} \frac{p-1}{p}\left(1+\frac{1}{p}\right)=\prod_{p>s}\left(1-\frac{1}{p^{2}}\right) \\
& >\prod_{n>s}\left(1-\frac{1}{n^{2}}\right)=\prod_{n>s} \frac{(n-1)(n+1)}{n^{2}}=\frac{s}{s+1},
\end{aligned}
$$

we see that taking $s>\frac{1-\epsilon / 2}{\epsilon / 2}=\frac{2-\epsilon}{\epsilon}$ suffices.

Second, we need to compute $\overline{\alpha_{s}}$ to sufficient accuracy so that the trailing terms in our series of reciprocals of $s$-smooth numbers is less than $\epsilon / 2$. Let

$$
N=\left\lceil-\log _{2}\left(\frac{\epsilon}{2 \pi(s)}\right)\right\rceil .
$$

Then

$$
\sum_{i>N} \frac{1}{p^{i}} \leq \sum_{i>N} \frac{1}{2^{i}}=\frac{1}{2^{N}} \leq \frac{\epsilon}{2 \pi(s)} .
$$

Then the error in approximating $\overline{\alpha_{s}}$ by using the $(N+1)^{\pi(s)} s$-smooth integers $B_{N}^{s}=\left\{2^{i_{1}} 3^{i_{2}} \cdots p_{\pi(s)}^{i_{\pi(s)}}: 0 \leq i_{k} \leq N\right\}$ is less than

$$
\begin{aligned}
\left(\prod_{p \leq s} \frac{p-1}{p}\right) \sum_{\substack{n \text { is } \\
\text { ismooth } \\
n \notin B_{N}^{s}}} \frac{1}{n} & <\left(\prod_{p \leq s} \frac{p-1}{p}\right) \sum_{\substack{p \leq s \\
n}} \sum_{\substack{\text { is } \\
v_{p}(n)>N}} \frac{1}{n} \\
& =\left(\prod_{p \leq s} \frac{p-1}{p}\right) \sum_{p \leq s}\left(\left(\sum_{i>N} \frac{1}{p^{i}}\right) \prod_{\substack{q \neq p \\
q \leq s}} \sum_{i \geq 0} \frac{1}{q}\right) \\
& =\sum_{p \leq s}\left(\frac{p-1}{p}\left(\sum_{i>N} \frac{1}{p^{i}}\right)\right)<\sum_{p \leq s} \frac{\epsilon}{2 \pi(s)}=\frac{\epsilon}{2}
\end{aligned}
$$

Now, using the $(N+1)^{\pi(s)}$ smallest $s$-smooth integers, rather than those in $B_{N}^{s}$, will only make the error smaller. So it will suffice for our computation to work with the exclusions required among the first $(N+1)^{\pi(s)} s$-smooth integers. In particular, we need to calculate for each $j \leq K$ the minimal number of exclusions required from the set of integers $S_{j}^{(s)}$.

We need to exclude at least one member of each three-term geometric progression contained in this set, an example of a 3-hitting set problem, which is a problem known to be NP-complete. In [20] Wahlström gives an algorithm for computing a 3 -hitting set for a set of size $n$ (and any collection of 3-element subsets of it) in time $O\left(1.6538^{n}\right)$. (He also gives an algorithm that requires exponential space but runs in time $O\left(1.6318^{n}\right)$.)

Using this algorithm for each $j \leq K$ to compute the minimal number of exclusions will require time $O\left(\sum_{j \leq K} 1.6538^{j}\right)=O\left(1.6538^{K}\right)$ in total. Substituting in 
the definitions of $K$ and $N$, we see this takes time

$$
O\left(1.6538\left(\left[-\log _{2}\left(\frac{\epsilon}{2 \pi\left(\frac{2-\epsilon}{\epsilon}\right)}\right)\right]+1\right)^{\pi\left(\frac{2-\epsilon}{\epsilon}\right)}\right)=O\left(1.6538^{\left.\log _{2}\left(\frac{8 \pi\left(\frac{2-\epsilon}{\epsilon}\right)}{\epsilon}\right)^{\pi\left(\frac{2-\epsilon}{\epsilon}\right)}\right)}\right)
$$

or, using the crude inequality $\pi\left(\frac{2-\epsilon}{\epsilon}\right)<8 \pi\left(\frac{2-\epsilon}{\epsilon}\right)<\frac{1}{\epsilon}$ for sufficiently small $\epsilon$,

$$
O\left(1.6538^{\left(-2 \log _{2} \epsilon\right)^{\frac{1}{\epsilon}}}\right) \text {. }
$$

Let $n_{1}, n_{2}, \ldots, n_{l}$ be the exclusion points returned by Wahlstrom's algorithm and set

$$
M=\prod_{p \leq s} \frac{p-1}{p} \sum_{i=1}^{l} \frac{1}{n_{i}} .
$$

We see that

$$
\begin{aligned}
1-M>\bar{\alpha} & >\left(1-M-\frac{\epsilon}{2}\right) \prod_{p>s}\left(\frac{p-1}{p} \sum_{i \in A_{3}^{*}} p^{-i}\right) \\
& >\left(1-M-\frac{\epsilon}{2}\right)\left(1-\frac{\epsilon}{2}\right)>1-M-\epsilon
\end{aligned}
$$

and thus we have achieved the required precision in our estimate of $\bar{\alpha}$.

The same arguments apply for the constant $\bar{\beta}$ as well. While it appears from our computations so far that the argument of Theorem 3.2 is far more efficient at computing lower bounds for $\bar{\beta}$ than the analogous argument to that described here for integer-ratios, it is not clear that the construction described there converges to $\bar{\beta}$.

\section{Sets With ASYMPtotic Density}

All of the upper bounds given here and elsewhere in the literature are for the upper density of geometric-progression-free sets, while the set Rankin constructed to produce a lower bound has an asymptotic density. It is quite possible that the restricted collection of such sets which possess an asymptotic density will have smaller densities.

We can prove that this is the case when avoiding progressions involving only one prime. In fact, we show this under the weaker hypothesis that such a set has logarithmic density. Any set, $S$, with asymptotic density also has logarithmic density equal to its asymptotic one,

$$
d(S)=\lim _{x \rightarrow \infty} \frac{1}{\log x} \sum_{\substack{s \in S \\ s \leq x}} \frac{1}{s} .
$$

We show here that any set with logarithmic density (and hence any set with asymptotic density) free of geometric progressions involving powers of 2 will have density strictly smaller than $\overline{\alpha_{2}} \approx 0.846378$, the supremum of the upper densities of all 2-smooth geometric-progression-free sets mentioned at the end of Section 4 . 
Theorem 6.1. The set, $T=\left\{n \in \mathbb{N}: v_{2}(n) \in A_{3}^{*}\right\}$ (where $A_{3}^{*}$ is the set free of arithmetic progressions obtained by the greedy algorithm), has the largest density among all sets which are free of 2-smooth-geometric progressions (of length 3) and have a logarithmic density. This set, $T$, has asymptotic density, $d(T)=$ $\frac{1}{2} \sum_{i \in A_{3}^{*}} 2^{-i} \approx 0.845398<\overline{\alpha_{2}}$.

Proof. The inclusion of any integer, $s$, in a set, $S$, avoiding 2-smooth geometric progressions only affects the possible inclusion of terms of the form $2^{i} s$ later in the set. Now, because

$$
\frac{1}{s}=\sum_{i>0} \frac{1}{2^{i} s}
$$

we see that the contribution of any term, $s$, to the sum in the logarithmic density of $S$ is greater than any possible contribution from later terms in the progression of multiples of $s$ by powers of 2 . The greedy set is thus seen to be optimal in this case: The contribution to the logarithmic density of any integer, $s$, outweighs any potential benefit which could be gained by excluding it.

The set $T$ is the set obtained by the greedy algorithm in this case. Each integer $t$ is included in $T$ if doing so does not create a 2 -smooth geometric progression with smaller integers already in $T$, which occurs if and only if the exponent of the greatest power of 2 dividing $t$ is in the set $A_{3}^{*}$.

This argument is readily seen to generalize to sets avoiding geometric progressions with ratios involving any single prime, or even to ratios composed of any fixed set of primes $P$ with the property that $\prod_{p \in P} \frac{p}{p-1} \leq 2$. In each case the greedy set is seen to be optimal among sets which have logarithmic (asymptotic) density and avoid geometric progressions with such ratios.

\section{Open questions}

This paper answers one of the questions in [13] by demonstrating a method of effectively computing the value of $\bar{\alpha}$. The question posed in that paper asks for the maximal upper density of sets avoiding progressions of arbitrary length, $k$. Our constant, $\bar{\alpha}$, is defined in regards to progressions of length 3 , but the methods here easily generalize to progressions of any length, $k$. Their second question, however, regarding the precise value of $\alpha$ remains open. We do not even know the answer to the following question.

Question 7.1. Is $\alpha$ strictly smaller than $\bar{\alpha}$ ?

This seems almost certain to be true, especially given the result of Section 6. In light of this result, and the computations required for Theorem 3.3 we make the stronger conjecture.

Conjecture 7.2. Rankin's set, $G_{3}^{*}$ has the largest possible density among geometricprogression-free sets which have an asymptotic density, so $\alpha=d\left(G_{3}^{*}\right)$.

One can ask the same question about $\beta$.

Question 7.3. Is $\beta<\bar{\beta}$ ?

Question 7.4. While we know from Theorem 3.3 that $\beta>d\left(G_{3}^{*}\right)$, and from Section 4 that $\bar{\beta}>\bar{\alpha}$, do we have $\beta>\alpha$ ? 
In [1] and [2] Beiglböck, Bergelson, Hindman, and Strauss take a more Ramseytheoretic view of the problem. They observe, for example, that a van der Waerden type theorem holds for geometric progressions: For any $k$-coloring of the natural numbers, there are arbitrarily long monochromatic integral ratio geometric progressions. One of the questions in [1] can be answered with the methods of their paper. They ask if there is a set $A$ of positive integers, free of three-term rational-ratio geometric progressions, such that $A$ has positive upper density and $A$ contains arbitrarily long intervals. Such a set can be constructed by alternating between long runs from Rankin's set of positive density, long gaps with no integers, and consecutive integers in an interval of the shape $[x, x+\sqrt{x}-1]$. Their Lemma 3.3 implies such an interval of consecutive integers has no three-term rational-ratio geometric progressions. Modifying this slightly, the set can even be taken to have positive lower density. Thanks are due to Carl Pomerance for these observations. Here is a nice problem from [1] that remains unsolved.

Question 7.5. Must every infinite set of natural numbers with bounded gaps between consecutive terms contain arbitrarily long geometric progressions?

\section{ACKNOWLEDGMENTS}

The author would like to thank Kevin Ford for suggesting the problem of geometric progressions in $\mathbb{Z} / n \mathbb{Z}$ and for allowing me to include his improvement, Theorem 3.2. The author would also like to thank his advisor, Carl Pomerance, for his support and invaluable guidance during the development of this paper and the referee for many useful suggestions.

\section{REFERENCES}

[1] M. Beiglböck, V. Bergelson, N. Hindman, and D. Strauss, Multiplicative structures in additively large sets, J. Combin. Theory Ser. A 113 (2006), no. 7, 1219-1242, DOI 10.1016/j.jcta.2005.11.003. MR.2259058(2007f:05174)

[2] M. Beiglböck, V. Bergelson, N. Hindman, and D. Strauss, Some new results in multiplicative and additive Ramsey theory, Trans. Amer. Math. Soc. 360 (2008), no. 2, 819-847, DOI 10.1090/S0002-9947-07-04370-X. MR2346473 (2008g:05214)

[3] T. F. Bloom, A quantitative improvement for Roth's theorem on arithmetic progressions, arXiv preprint arXiv:1405.5800 (2014).

[4] J. Bourgain, On triples in arithmetic progression, Geom. Funct. Anal. 9 (1999), no. 5, 968984, DOI 10.1007/s000390050105. MR.1726234(2001h:11132)

[5] B. E. Brown and D. M. Gordon, On sequences without geometric progressions, Math. Comp. 65 (1996), no. 216, 1749-1754, DOI 10.1090/S0025-5718-96-00765-X. MR.1361804 (97a:11024)

[6] P. Erdős, C. Pomerance, and E. Schmutz, Carmichael's lambda function, Acta Arith. 58 (1991), no. 4, 363-385. MR.1121092 (92g:11093)

[7] J. B. Friedlander, C. Pomerance, and I. E. Shparlinski, Period of the power generator and small values of Carmichael's function, Math. Comp. 70 (2001), no. 236, 1591-1605 (electronic), DOI 10.1090/S0025-5718-00-01282-5. MR.1836921 (2002g:11112)

[8] G. H. Hardy and E. M. Wright, An Introduction to the Theory of Numbers, 5th ed., The Clarendon Press, Oxford University Press, New York, 1979. MR568909 (81i:10002)

[9] D. R. Heath-Brown, Integer sets containing no arithmetic progressions, J. London Math. Soc. (2) 35 (1987), no. 3, 385-394, DOI 10.1112/jlms/s2-35.3.385. MR889362 (88g:11005)

[10] V. F. Lev, Progression-free sets in finite abelian groups, J. Number Theory 104 (2004), no. 1, 162-169, DOI 10.1016/S0022-314X(03)00148-3. MR2021632(2004k:11023)

[11] R. Meshulam, On subsets of finite abelian groups with no 3-term arithmetic progressions, J. Combin. Theory Ser. A 71 (1995), no. 1, 168-172, DOI 10.1016/0097-3165(95)90024-1. MR 1335785 (96g:20033) 
[12] M. B. Nathanson and K. O'Bryant, Irrational numbers associated to sequences without geometric progressions, Integers 14 (2014), Paper No. A40, 11 pp. MR.3247169

[13] M. B. Nathanson and K. O'Bryant, On sequences without geometric progressions, Integers 13 (2013), Paper No. A73, 5. MR3141833

[14] R. A. Rankin, Sets of integers containing not more than a given number of terms in arithmetical progression, Proc. Roy. Soc. Edinburgh Sect. A 65 (1960/1961), 332-344 (1960/61). MR0142526 (26 \#95)

[15] J. Riddell, Sets of integers containing no $n$ terms in geometric progression, Glasgow Math. J. 10 (1969), 137-146. MR.0257022 (41 \#1677)

[16] K. Roth, Sur quelques ensembles d'entiers (French), C. R. Acad. Sci. Paris 234 (1952), 388-390. MR0046374(13,724d)

[17] K. F. Roth, On certain sets of integers, J. London Math. Soc. 28 (1953), 104-109. MR0051853 $(14,536 \mathrm{~g})$

[18] T. Sanders, On Roth's theorem on progressions, Ann. of Math. (2) 174 (2011), no. 1, 619-636, DOI 10.4007/annals.2011.174.1.20. MR2811612 (2012f:11019)

[19] E. Szemerédi, Integer sets containing no arithmetic progressions, Acta Math. Hungar. 56 (1990), no. 1-2, 155-158, DOI 10.1007/BF01903717. MR.1100788 (92c:11100)

[20] M. Wahlström, Exact algorithms for finding minimum transversals in rank-3 hypergraphs, J. Algorithms 51 (2004), no. 2, 107-121, DOI 10.1016/j.jalgor.2004.01.001. MR2050139 (2005a:05185)

Department of Mathematics, Dartmouth College, Hanover, New Hampshire 03755

E-mail address: nathan.g.mcnew.gr@dartmouth.edu 